\title{
CARACTERIZACIÓN TÉRMICA DE MEZCLAS DE ARCILLAS UTILIZADAS EN LA FABRICACIÓN DE PRODUCTOS DE MAMPOSTERÍA PARA LA CONSTRUCCIÓN
}

\section{THERMAL CHARACTERIZATION OF MIXES OF CLAYS USED IN THE MANUFACTURE OF MASONRY PRODUCTS FOR CONSTRUCTION}

\author{
MSc. Ricardo Andrés García-León", MSc. Eder Flórez Solano** \\ PhD. Carlos Acevedo Peñaloza **** \\ *Universidad Francisco de Paula Santander Ocaña, \\ Facultad de Ingenierías, Grupo de Investigación INGAP. \\ Vía Acolsure, Sede el Algodonal - Ocaña, Norte de Santander, Colombia. \\ Tel.: +57 5690088 Ext. 212 - 191. \\ E-mail: (ragarcial, enflorez) @ufpso.edu.co. \\ ${ }^{* *}$ Universidad Francisco de Paula Santander, \\ Facultad de Ingenierías, programa de ingeniería Mecánica. \\ Cúcuta, Norte de Santander. \\ E-mail: carloshumbertoap@ufps.edu.co.
}

\begin{abstract}
Resumen: Actualmente en Norte de Santander existe gran variedad de yacimientos naturales que se pueden utilizar para fabricar productos de mampostería para la construcción como lo son bloques, tejas, ladrillos, baldosas, entre otros; pero las empresas fabricantes obtienen muchos desperdicios debido a la falta de análisis tecnológicos de la materia prima para pronosticar el comportamiento de las pastas cerámicas y lograr mejorar la calidad del producto final. En el presente trabajo se realizó las caracterizaciones térmicas de dos mezclas de arcillas ( $\mathrm{m} 7 \mathrm{y} \mathrm{m} 8)$ encontradas experimentalmente utilizando el método de moldeo de extrusión y en polvo para la fabricación de bloques H-10 tomando como muestra la materia prima (arcilla) de una empresa dedicada a la fabricación de bloques H-10 en Ocaña, Norte de Santander. El desarrollo de la investigación se llevó a cabo mediante la ejecución de ensayos térmicos a las muestras de arcillas con los que se determinaron los porcentajes pérdida de peso respecto a las temperaturas obtenidas en un diagrama con las curvas de ATD y ATG. Los resultados obtenidos demuestran que las mezcla $\mathrm{m} 7$ encontrada experimentalmente presenta mejores características en el proceso de cocción por lo que se estableció una curva de cocción óptima para la fabricación del bloque H-10 teniendo en cuenta las especificaciones del proceso productivo y la materia prima, con lo que se espera mejorar la calidad del producto final cumpliendo las especificaciones de las normas actuales vigentes. Es indispensable caracterizar las arcillas para optimizar la pasta de producción y evitar imperfecciones en el producto final (Bloque H-10) con lo que evidentemente se mejoraran los recursos ambientales y económicos de la empresa.
\end{abstract}

Palabras clave: Térmica, ATG, ATD, Arcillas, Bloque H-10.

Abstract: Currently in Norte de Santander there is a great variety of natural deposits that can be used to manufacture masonry products for construction such as blocks, tiles, bricks, tiles, among others; But the manufacturers obtain a lot of waste due to the lack of technological analysis of the raw material to forecast the behavior of the ceramic pastes 
and to improve the quality of the final product. In the present work, the thermal characterizations of two mixtures of clays $(\mathrm{m} 7$ and $\mathrm{m} 8$ ) experimentally found using the extrusion and powder molding method for the manufacture of H-10 blocks were made taking as sample the raw material (clay) of a company dedicated to the manufacture of $\mathrm{H}$ 10 blocks in Ocaña, Norte de Santander. The development of the investigation was carried out by means of the execution of thermal tests to the samples of clays with which the percentages of weight loss were determined with respect to the temperatures obtained in a diagram with the ATD and ATG curves. The results obtained show that the $\mathrm{m} 7$ mixture found experimentally has better characteristics in the cooking process, so an optimal cooking curve was established for the manufacture of the H-10 block taking into account the specifications of the production process and the raw material, with what is expected to improve the quality of the final product meeting the specifications of the current regulations in force. It is essential to characterize the clays to optimize the production paste and avoid imperfections in the final product (Block $\mathrm{H}-10$ ), which evidently will improve the company's environmental and economic resources.

Keywords: Publishing rules, procedures, publication, IFAC format.

\section{INTRODUCCIÓN}

El proceso de transformación de la arcilla se compone principalmente de tres fases, que son; preparación de la pasta cerámica, moldeo de la pieza y cocción. El proceso de preparación es la primera fase, en donde se modifica la composición y la plasticidad adicionando otras arcillas de la región con la finalidad de obtener una pasta cerámica homogénea, en la segunda fase de moldeo se debe tener en cuenta la forma deseada si se llevará a cobo a presión o extrusión. Una vez se ha obtenido la pieza moldeada, se procede al secado que es la última fase, en donde se controla la velocidad de evaporación de agua con el fin de evitar defectos en la pieza. Posteriormente, la pieza se sinteriza, es decir, se somete al proceso de cocción con el fin disminuir la porosidad, aumentar la densidad y la resistencia mecánica (Muñoz Meneses R. A., Muñoz Chaves J.A., Mancilla P, 2007) (Barranzuela, 2014) (Jácome Manzano, 2012) (García-León \& Flórez Solano, 2016) (García-León, Bolívar León, \& Flórez Solano, 2016) (Velasco Sánchez, Sánchez Lozano, Peral Orts, \& AME, 2016).

De acuerdo con su capacidad de producción y desarrollo tecnológico las industrias ladrilleras se han clasificado en chircales, ladrilleras pequeñas, medianas y grandes (Riojas Castillo \& Rodríguez Montaña, 2004). Por lo general, estas industrias mantienen el proceso productivo como se puede observar en la figura 1.

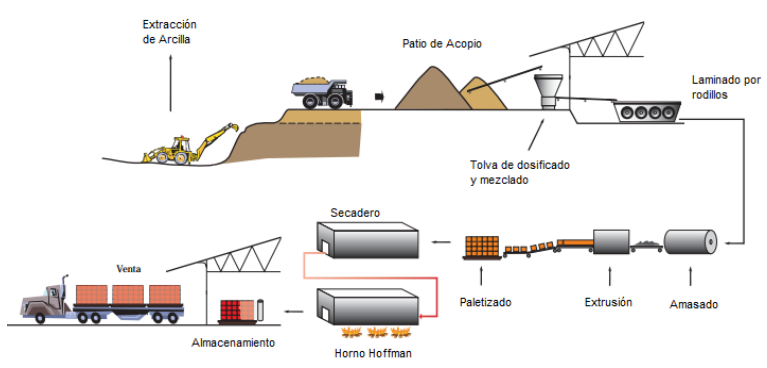

Figura 1. Proceso de producción de una ladrillera.

Fuente: (Rodríguez, 2007)

Es importante realizar la identificación del proceso y de las variables que intervienen en cada una de sus etapas del proceso productivo como la investigación realizada por (García-León \& Flórez Solano, 2016). Como también la caracterización de la materia prima (la arcilla) por diferentes métodos como: Ensayos físicos, químicos y mecánicos, con la finalidad de conocer el comportamiento en las diferentes etapas de producción y como validar el producto final con condiciones de calidad basadas en las normas actuales vigentes como la NTC 4017 y 4205 que rigen los productos de mampostería para la construcción (García-León et al., 2016)(García-León \& Bolívar León, 2017).

En una investigación realizada por (Carriazo, Molina, \& Moreno, 2008) se realizó el análisis termoravimetrico por TGA y DTA y demás ensayos para evaluar el comportamiento de las arcillas con soluciones de Al-Ce-Fe.

Otros estudios analizaron una muestra de arcilla roja y determinaron su composición química y las 
fases cristalinas; además de la distribución granulométrica y análisis térmicos también se determinó, la densidad y los límites de Atterberg; donde posteriormente evaluaron las muestras térmicamente a $1050^{\circ} \mathrm{C}$, además de análisis químicos como DRX y FRX (Zuluaga Castrillón et al., 2016) (Perales López \& Barrera Vargas, 2013) (Cely-Illera, 2016). Siendo de gran importancia para la industria de mampostería para la construcción este tipo de análisis de laboratorio para poder pronosticar el comportamiento de las arcillas durante las etapas de producción.

\section{MATERIALES Y MÉTODOS}

Para el desarrollo de este trabajo se llevó a cabo una investigación con profundidad descriptiva, de diseño experimental, con un enfoque mixto (Cualitativo - Cuantitativo) de propósito básico. Teniendo en cuenta los análisis de laboratorio y las variables que intervinieron en la evaluación de los resultados (García-León, Flórez Solano, \& Acosta Pérez, 2015) (Araque, Díaz, \& Gualdrón, 2012)(Moreno Rubio, Jimenez López, \& Barrera Lombana, 2013) (Flórez Solano, García-León, \& Escrobar Macea, 2017) (Mejía, Augusto, Cortés, Ivan, \& Jaimes, 2016).

El análisis térmico diferencial ATD, mide la diferencia de temperatura entre la muestra y el material de referencia respecto a la temperatura alcanzada en cada instante. Estas medidas se pueden realizar en condiciones ambientales o bajo una atmósfera controlada, con el fin de determinar los efectos endotérmicos y exotérmicos que acompañan a las reacciones de la muestra en la cocción (Douglas, Skoog, Holler, \& Timothy A, 2001) (Rendon Ríos, 2009) (Sáenz García, 2014).

Por otra parte, el análisis termogravimétrico ATG, es una técnica en la que la masa de la muestra es controlada a una temperatura programada, con lo que se puede determinar la pérdida de peso de las muestras que son generadas en la etapa de cocción, es decir la descomposición térmica de los materiales. Este análisis se complementa con los análisis obtenidos en el ATD (Linseis., 2012) (Ramírez Calderón, 2012) (Florez Arenas, 2015) (Cely Illera \& Bolívar León, 2015).

La caracterización térmica se llevó a cabo para determinar las posibles variaciones en cuanto a dimensiones y características morfológicas de las dos mezclas optimas obtenidas experimentalmente en otras fases de la investigación, mediante los análisis térmicos diferencial (ATD) y análisis termogravimétrico (ATG), a través una termobalanza Q600 de alta sensibilidad.

Los estudios se realizaron sobre dos muestras sólidas conformadas por el método de moldeo de extrusión y otra en polvo de las mezclas denominadas $\mathrm{m} 7$ y $\mathrm{m} 8$ experimentales en el laboratorio para la fabricación de bloques H-10 perforados horizontalmente, en un rango de temperatura entre $20^{\circ} \mathrm{C}$ y $1200^{\circ} \mathrm{C}$ en presencia de una atmosfera controlada de oxígeno y en un ciclo térmico de $5^{\circ} \mathrm{C} / \mathrm{min}$. Las curvas ATD y ATG obtenidas permitieron observar los picos endotérmicos y exotérmicos acompañado de las pérdidas de peso que sufre el material, utilizando el software Data Analysis que posee el equipo para el ensayo (Guerrero Gomez, Marrugo Carreazo, \& Gomez Camperos, 2015).

Para el evaluar el comportamiento de las muestras analizadas, se tuvo en cuenta la siguiente figura 2 la cual representa las curvas características de los análisis de termogravimétria.

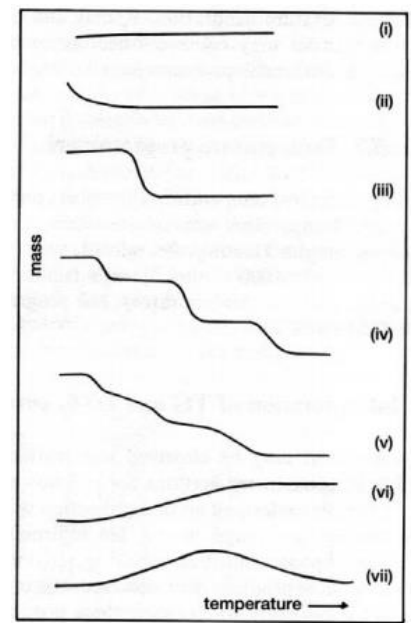

Figura 2. Principales tipos de curvas termogravimétricas.

Fuente: (Sanchez, 2016)

Cada una de las curvas características anteriores descritas en la figura 2, describen el siguiente comportamiento:

Tipo (i). La muestra no sufre descomposición con pérdida de productos volátiles en el rango de temperatura mostrado. Pudiera ocurrir reacciones tipo: transición de fase, fundido, polimerización.

Tipo (ii). Una rápida pérdida de masa inicial es característica de procesos de desorción o secado.

Tipo (iii). Esta curva representa la descomposición de la muestra en un proceso simple. La curva se puede utilizar para definir los límites de estabilidad 
del reactante, determinar la estequiometría e investigar la cinética de las reacciones.

Tipo (iv). Se indica una descomposición multietapa con intermedios relativamente estables. Se puede definir los límites de estabilidad del reactante e intermedios, y de forma más compleja la estequiometría la reacción.

Tipo (v). También indica una descomposición multietapa, pero los productos intermedios no son estables, y poca información se obtiene de la estequiometría de la reacción.

Tipo (vi). Se observa una ganancia de masa como consecuencia de la reacción de la muestra con la atmósfera que la rodea.

Tipo (vii). El producto de una reacción de oxidación se descompone a temperaturas más elevadas: $2 \mathrm{Ag}+1 / 2 \mathrm{O} 2 Æ \mathrm{Ag} 2 \mathrm{O} \rightleftarrows 2 \mathrm{Ag}+1 / 2 \mathrm{O} 2$.

\section{RESULTADOS Y DISCUSIÓN}

Luego de realizar el montaje de las muestras conformadas $y$ en polvo en el equipo de laboratorio, a continuación en la figura 3 y 4 , se presentan los resultados del análisis térmico para la mezcla m7:

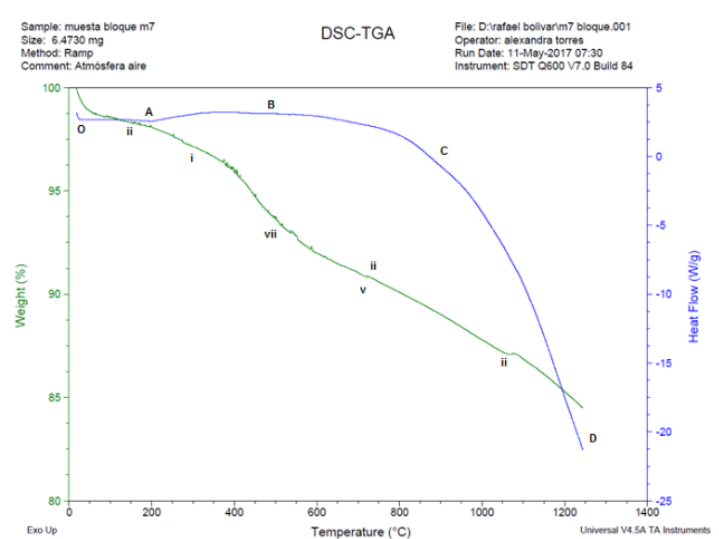

Figura 3. Termograma de los análisis térmicos de ATD y ATG, para la mezcla $m 7$ - muestra conformada.

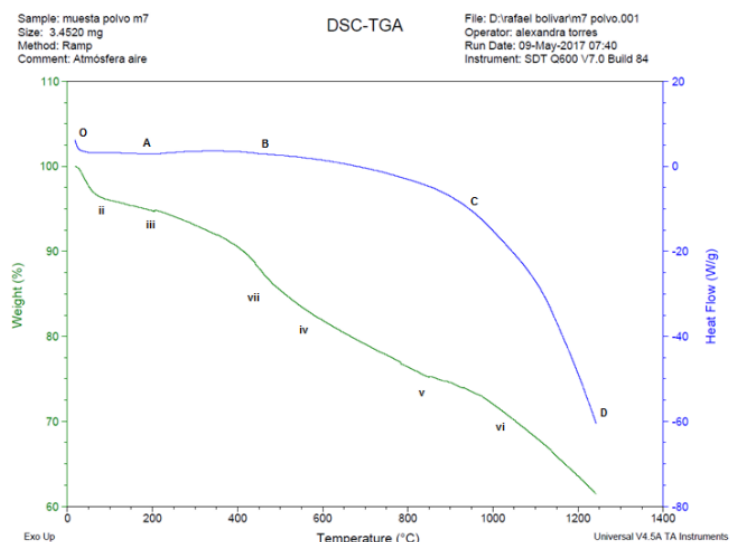

Figura 4. Termograma de los análisis térmicos de ATD y ATG, para la mezcla $m 7$ - muestra en polvo.

Para las curvas las figuras anteriores, se observa el siguiente comportamiento teniendo en cuenta las curvas características:

Tabla 1. Análisis de las gráficas ATD y ATG para la mezcla $m 7$.

\begin{tabular}{|c|c|c|c|c|}
\hline \multirow{3}{*}{$\mathbf{T}^{\circ} \mathbf{C}$} & \multicolumn{4}{|c|}{ Curva } \\
\hline & \multicolumn{2}{|c|}{ ATD (azul) } & \multicolumn{2}{|c|}{ ATG (verde) } \\
\hline & Figura 1 & Figura 2 & Figura 1 & Figura2 \\
\hline $0-50$ & $\begin{array}{l}\text { Cambio } \\
\text { endotérmic } \\
\text { asociada } \\
\text { de deso } \\
\text { secado. } \\
\text { puntos O- }\end{array}$ & $\begin{array}{l}\text { o, } \\
\text { procesos } \\
\text { rción } \quad 0 \\
\text { ntre los }\end{array}$ & $\begin{array}{l}\text { Pérdida de peso } \\
\text { del 1,25\% } \\
\text { relacionada a la } \\
\text { pérdida de agua } \\
\text { higroscópica, } \\
\text { libre o la } \\
\text { humedad } \\
\text { residual de la } \\
\text { muestra. Se } \\
\text { presenta una } \\
\text { curva } \\
\text { característica } \\
\text { tipo II. }\end{array}$ & $\begin{array}{l}\text { Pérdida de peso } \\
\text { del } 2,60 \% \\
\text { relacionada a la } \\
\text { pérdida de agua } \\
\text { higroscópica, libre } \\
\text { o la humedad } \\
\text { residual de la } \\
\text { muestra. } \\
\text { presenta una curva } \\
\text { característica tipo } \\
\text { II. }\end{array}$ \\
\hline $\begin{array}{l}50- \\
200\end{array}$ & $\begin{array}{l}\text { Entre los } \\
\text { A, se ap } \\
\text { ligera esta } \\
\text { debido a } \\
\text { generan } \\
\text { químicas. }\end{array}$ & $\begin{array}{l}\text { puntos O- } \\
\text { recia una } \\
\text { illización, } \\
\text { jue no se } \\
\text { eacciones }\end{array}$ & $\begin{array}{l}\text { Se presenta una } \\
\text { pérdida de masa } \\
\text { de } 1,30 \% \text {, } \\
\text { producto de la } \\
\text { evaporación de } \\
\text { agua, ligada a } \\
\text { las estructuras } \\
\text { de las muestras. }\end{array}$ & $\begin{array}{l}\text { Se presenta una } \\
\text { pérdida de masa } \\
\text { de } 2,40 \% \text {, } \\
\text { producto de la } \\
\text { evaporación de } \\
\text { agua, ligada a las } \\
\text { estructuras de las } \\
\text { muestras. Se } \\
\text { presenta una curva } \\
\text { característica tipo } \\
\text { III. }\end{array}$ \\
\hline $\begin{array}{l}200- \\
450\end{array}$ & $\begin{array}{l}\text { Entre los } \\
\mathrm{B} \text {, se pro } \\
\text { reacción e } \\
\text { Por la } \\
\text { oxidante } \\
\text { hidróxidos } \\
\left(\mathrm{Fe}_{2} \mathrm{O}_{3}\right) \text {. }\end{array}$ & $\begin{array}{l}\text { buntos A- } \\
\text { dujo una } \\
\text { xotérmica. } \\
\text { isociación } \\
\text { de los } \\
\text { de hierro }\end{array}$ & $\begin{array}{l}\text { Se presenta una } \\
\text { pérdida de masa } \\
\text { de } 3,5 \% \text {. Entre } \\
\text { los } 250^{\circ} \mathrm{C} \\
\text { aparece la curva } \\
\text { característica } \\
\text { tipo I } \\
\text { relacionada con } \\
\text { la presencia de } \\
\text { montmorillonita } \\
\text { (desorción de } \\
\text { agua). }\end{array}$ & $\begin{array}{l}\text { Se presenta una } \\
\text { pérdida de masa } \\
\text { de } 7,5 \% \text {. Entre los } \\
440^{\circ} \mathrm{C} \text { aparece la } \\
\text { curva } \\
\text { característica tipo } \\
\text { VII relacionada } \\
\text { con la presencia } \\
\text { de } \\
\text { montmorillonita } \\
\text { (desorción de } \\
\text { agua). }\end{array}$ \\
\hline $\begin{array}{r}400- \\
650\end{array}$ & $\begin{array}{l}\text { Entre } \\
\text { los } \\
\text { puntos } \\
\text { B-C, se } \\
\text { observa } \\
\text { una } \\
\text { reacción }\end{array}$ & $\begin{array}{l}\text { En el } \\
\text { puntos } \\
\mathrm{B}, \quad \text { se } \\
\text { inicia } \\
\text { una } \\
\text { reacción } \\
\text { endotér }\end{array}$ & $\begin{array}{l}\text { Se presenta la } \\
\text { expulsión del } \\
\text { agua } \\
\text { cristalizada, } \\
\text { como también } \\
\text { la curva } \\
\text { característica } \\
\end{array}$ & $\begin{array}{l}\text { Se presenta la } \\
\text { expulsión del agua } \\
\text { cristalizada, como } \\
\text { también la curva } \\
\text { característica tipo } \\
\text { IV y VII, con una } \\
\text { pérdida de peso }\end{array}$ \\
\hline
\end{tabular}




\begin{tabular}{|c|c|c|c|}
\hline & \begin{tabular}{l|l} 
exotérm & mica \\
ica & con un \\
relacion & pequeño \\
ada con & salto \\
la & relacion \\
transfor & ada con \\
mación & la \\
alotrópi & transfor \\
ca del & mación \\
cuarzo $\alpha$ & alotrópi \\
a $\beta$, & ca del \\
común a & cuarzo $\alpha$ \\
temperat & a $\beta$, \\
uras de & común a \\
$573^{\circ} \mathrm{C}$. & temperat \\
& uras de \\
& $573^{\circ} \mathrm{C}$.
\end{tabular} & $\begin{array}{l}\text { tipo VII, con } \\
\text { una pérdida de } \\
\text { peso del } 3,75 \%\end{array}$ & del $11 \%$ \\
\hline $\begin{array}{l}600- \\
1050\end{array}$ & $\begin{array}{l}\text { En el punto } \mathrm{C} \text { a los } \\
950^{\circ} \mathrm{C} \\
\text { aproximadamente se } \\
\text { presenta la reacción } \\
\text { endotérmica, este } \\
\text { efecto podría } \\
\text { corresponder a la } \\
\text { deshidroxilación } \\
\text { (dilución de los } \\
\text { gases combustibles } \\
\text { por el agua formada) } \\
\text { de la arcilla que se } \\
\text { prolongaría hasta la } \\
\text { temperatura de } \\
\text { fusión. }\end{array}$ & $\begin{array}{l}\text { La curva } \\
\text { presenta un } \\
\text { descenso } \\
\text { gradual y } \\
\text { progresivo, con } \\
\text { curvas } \\
\text { características } \\
\text { de tipo II y V, } \\
\text { con una pérdida } \\
\text { de peso del } 5 \% \text {. } \\
\text { En donde se } \\
\text { pudo haber } \\
\text { presentado la } \\
\text { liberación de } \\
\mathrm{CO}_{2} \text {. }\end{array}$ & $\begin{array}{l}\text { La curva presenta } \\
\text { un descenso } \\
\text { gradual r y } \\
\text { progresivo, con } \\
\text { curvas } \\
\text { características de } \\
\text { tipo } \mathrm{V} \text { y } \mathrm{VI} \text {, con } \\
\text { una pérdida de } \\
\text { peso del } 12 \% \text {. En } \\
\text { donde se pudo } \\
\text { haber presentado } \\
\text { la liberación de } \\
\mathrm{CO}_{2} \text {. }\end{array}$ \\
\hline $\begin{array}{l}1050 \\
- \\
1250\end{array}$ & $\begin{array}{l}\text { Entre los puntos C- } \\
\mathrm{D} \text {, se observa el } \\
\text { descenso progresivo } \\
\text { constante de la } \\
\text { curva, } \\
\text { probablemente a que } \\
\text { el material ya se } \\
\text { haya fusionado, la } \\
\text { cual no se estabiliza } \\
\text { debido a que la fase } \\
\text { encontrada para la } \\
\text { muestra m7 está a } \\
\text { los } 1500^{\circ} \mathrm{C} \\
\text { aproximadamente } \\
\text { que es donde se } \\
\text { obtiene mullita. La } \\
\text { cual se comienza a } \\
\text { formar a los } 980^{\circ} \mathrm{C} \\
\text { aproximadamente. }\end{array}$ & $\begin{array}{l}\text { Se presenta una } \\
\text { curva } \\
\text { característica de } \\
\text { tipo I, con una } \\
\text { pérdida de peso } \\
\text { del } 4 \% \text {. } \\
\text { En total se } \\
\text { obtiene a esta } \\
\text { temperatura una } \\
\text { pérdida de peso } \\
\text { del } 17 \%\end{array}$ & $\begin{array}{l}\text { Se presenta una } \\
\text { pérdida de peso } \\
\text { del } 7 \% \text {. } \\
\text { En total se obtiene } \\
\text { a esta temperatura } \\
\text { una pérdida de } \\
\text { peso del } 37 \%\end{array}$ \\
\hline
\end{tabular}

El inicio de la vitrificación aumenta la resistencia mecánica de la pieza, esta fase se obtiene a partir de $\operatorname{los} 1200^{\circ} \mathrm{C}$ (tabla 1) (Amado, Villafrades, \& Tuta, 2011). Además, no se aprecia una estabilización de la curva ATD porque la fase mullita se obtiene a $\operatorname{los} 1500^{\circ} \mathrm{C}$ aproximadamente, si se alcanzara esta temperatura, se obtendrían bloques refractarios. Ambos diagramas presentan un comportamiento similar, aunque se comporta mejor la muestra $\mathrm{m} 7$ en polvo.

A continuación en la figura 5 y 6 , se presentan los resultados del análisis para la mezcla m8 conformada y en polvo:

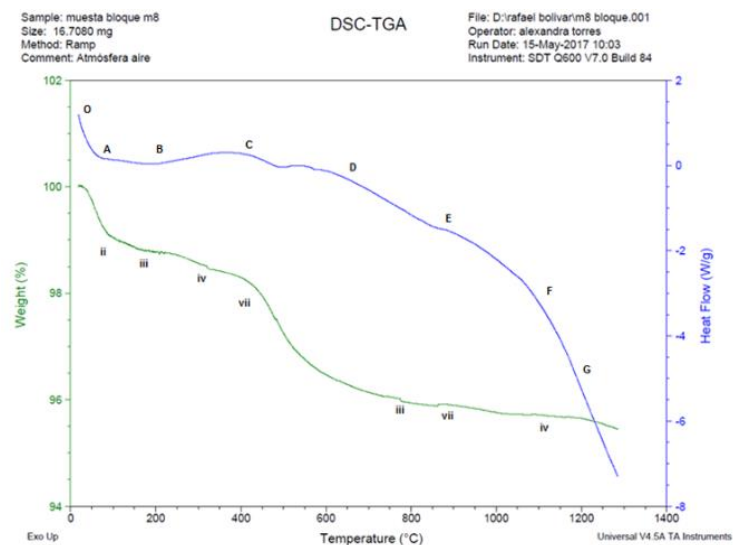

Figura 5. Termograma de los análisis térmicos de ATD y ATG, para la mezcla m8 - muestra conformada.

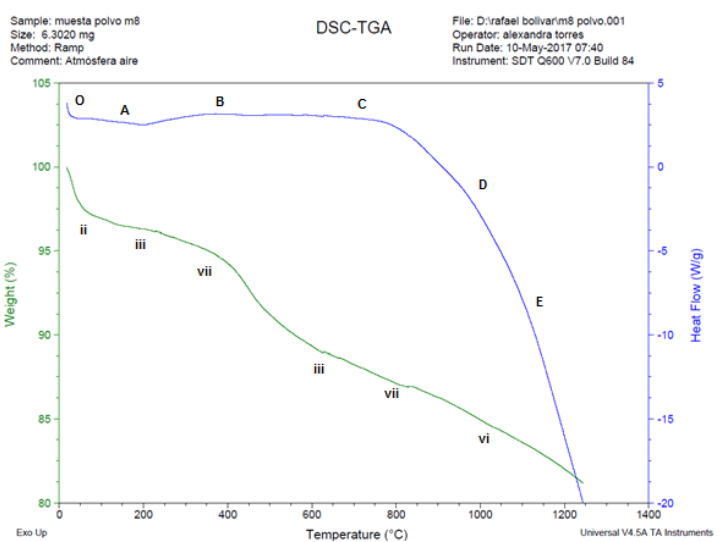

Figura 6. Termograma de los análisis térmicos de ATD y ATG, para la mezcla $m 8$ - muestra en polvo

Para las curvas las figuras anteriores, se observa el siguiente comportamiento teniendo en cuenta las curvas características:

Tabla 2. Análisis de las gráficas ATD y ATG para la mezcla $m 8$.

\begin{tabular}{|c|c|c|c|c|}
\hline \multirow{3}{*}{$\mathbf{T}^{\circ} \mathbf{C}$} & \multicolumn{4}{|c|}{ Curva } \\
\hline & \multicolumn{2}{|c|}{ ATD (azul) } & \multicolumn{2}{|c|}{ ATG (verde) } \\
\hline & Figura 5 & Figura 6 & Figura 5 & Figura 6 \\
\hline $0-50$ & $\begin{array}{l}\text { Se produce } \\
\text { endotérmico, } \\
\text { procesos de } \\
\text { secado. Entr } \\
\text { O-A. }\end{array}$ & $\begin{array}{l}\text { un cambio } \\
\text { asociada a } \\
\text { desorción o } \\
\text { los puntos }\end{array}$ & $\begin{array}{l}\text { Pérdida de } \\
\text { peso del } \\
0,9 \% \\
\text { relacionada a } \\
\text { la pérdida de } \\
\text { agua } \\
\text { higroscópica, } \\
\text { libre o la } \\
\text { humedad } \\
\text { residual de la } \\
\text { muestra. Se } \\
\text { presenta una } \\
\text { curva } \\
\text { característica } \\
\text { tipo II. }\end{array}$ & $\begin{array}{l}\text { Pérdida de peso } \\
\text { del } 2,5 \% \\
\text { relacionada a la } \\
\text { pérdida de agua } \\
\text { higroscópica, } \\
\text { libre o la } \\
\text { humedad } \\
\text { residual de la } \\
\text { muestra. Se } \\
\text { presenta una } \\
\text { curva } \\
\text { característica } \\
\text { tipo II. }\end{array}$ \\
\hline $\begin{array}{l}50- \\
200\end{array}$ & $\begin{array}{l}\text { Entre los } \\
\text { puntos } \\
\text { B, } \\
\begin{array}{ll}\text { aprecia } \\
\text { una ligera }\end{array} \\
\end{array}$ & $\begin{array}{ll}\text { Entre los } \\
\text { puntos } & \mathrm{O}- \\
\mathrm{A}, & \text { se } \\
\text { aprecia } & \\
\text { una ligera }\end{array}$ & $\begin{array}{l}\text { Se presenta } \\
\text { una pérdida } \\
\text { de masa de } \\
0,4 \% \text {, } \\
\text { producto de }\end{array}$ & $\begin{array}{l}\text { Se presenta una } \\
\text { pérdida de masa } \\
\text { de } 1,25 \% \text {, } \\
\text { producto de la } \\
\text { evaporación de }\end{array}$ \\
\hline
\end{tabular}




\begin{tabular}{|c|c|c|c|c|}
\hline & $\begin{array}{l}\text { estabilizaci } \\
\text { ón, debido } \\
\text { a que no se } \\
\text { generan } \\
\text { reacciones } \\
\text { químicas } \\
\text { importante } \\
\text { s. }\end{array}$ & $\begin{array}{l}\text { estabilizaci } \\
\text { ón, debido } \\
\text { a que no se } \\
\text { generan } \\
\text { reacciones } \\
\text { químicas } \\
\text { importante } \\
\text { s. }\end{array}$ & $\begin{array}{l}\text { la } \\
\text { evaporación } \\
\text { de agua, } \\
\text { ligada a las } \\
\text { estructuras de } \\
\text { las muestras. } \\
\text { Se presenta } \\
\text { una curva } \\
\text { característica } \\
\text { tipo III. }\end{array}$ & $\begin{array}{l}\text { agua, ligada a } \\
\text { las estructuras } \\
\text { de las muestras. } \\
\text { Se presenta una } \\
\text { curva } \\
\text { característica } \\
\text { tipo III. }\end{array}$ \\
\hline $\begin{array}{c}200- \\
450\end{array}$ & $\begin{array}{l}\text { Entre los } \\
\text { puntos B- } \\
\mathrm{C} \text {, se } \\
\text { produjo } \\
\text { una } \\
\text { reacción } \\
\text { exotérmica } \\
\text { Por la } \\
\text { disociació } \\
\text { n oxidante } \\
\text { de los } \\
\text { hidróxidos } \\
\text { de hierro } \\
\left(\mathrm{Fe}_{2} \mathrm{O}_{3}\right) .\end{array}$ & $\begin{array}{l}\text { Entre los } \\
\text { puntos A- } \\
\mathrm{B} \text {, se } \\
\text { produjo } \\
\text { una } \\
\text { reacción } \\
\text { exotérmica } \\
\text { Por la } \\
\text { disociació } \\
\text { n oxidante } \\
\text { de los } \\
\text { hidróxidos } \\
\text { de hierro } \\
\left(\mathrm{Fe}_{2} \mathrm{O}_{3}\right) .\end{array}$ & $\begin{array}{l}\text { Se presenta } \\
\text { una pérdida } \\
\text { de masa de } \\
0,8 \% \text {. Entre } \\
\text { los } 300^{\circ} \mathrm{C} \\
\text { aparece la } \\
\text { curva } \\
\text { característica } \\
\text { tipo IV } \\
\text { relacionada } \\
\text { con la } \\
\text { presencia de } \\
\text { montmorillon } \\
\text { ita (desorción } \\
\text { de agua). }\end{array}$ & $\begin{array}{l}\text { Se presenta una } \\
\text { pérdida de masa } \\
\text { de } 3,75 \% \text {. Entre } \\
\text { los } 350^{\circ} \mathrm{C} \\
\text { aparece la curva } \\
\text { característica } \\
\text { tipo VII } \\
\text { relacionada con } \\
\text { la presencia de } \\
\text { montmorillonita } \\
\text { (desorción de } \\
\text { agua). }\end{array}$ \\
\hline $\begin{array}{c}400- \\
650\end{array}$ & $\begin{array}{l}\text { Entre los } \\
\text { puntos C- } \\
D, \quad \text { se } \\
\text { observa } \\
\text { una } \\
\text { reacción } \\
\text { exotérmica } \\
\text { relacionad } \\
\text { a con la } \\
\text { transforma } \\
\text { ción } \\
\text { alotrópica } \\
\text { del cuarzo } \\
\alpha \quad \text { a } \beta, \\
\text { común a } \\
\text { temperatur } \\
\text { as de } \\
573^{\circ} \mathrm{C}\end{array}$ & $\begin{array}{l}\text { Entre los } \\
\text { puntos B- } \\
\text { C, se } \\
\text { observa } \\
\text { una } \\
\text { reacción } \\
\text { exotérmica } \\
\text { constante } \\
\text { relacionad } \\
\text { a con la } \\
\text { transforma } \\
\text { ción } \\
\text { alotrópica } \\
\text { del cuarzo } \\
\alpha \quad \text { a } \beta, \\
\text { común a } \\
\text { temperatur } \\
\text { as de } \\
573^{\circ} \mathrm{C}\end{array}$ & $\begin{array}{l}\text { Se presenta } \\
\text { la expulsión } \\
\text { del agua } \\
\text { cristalizada, } \\
\text { como } \\
\text { también la } \\
\text { curva } \\
\text { característica } \\
\text { tipo VII, con } \\
\text { una pérdida } \\
\text { de peso del } \\
2 \%\end{array}$ & $\begin{array}{l}\text { Se presenta la } \\
\text { expulsión del } \\
\text { agua } \\
\text { cristalizada, } \\
\text { como también } \\
\text { la curva } \\
\text { característica } \\
\text { tipo III, con una } \\
\text { pérdida de peso } \\
\text { del } 5,8 \%\end{array}$ \\
\hline $\begin{array}{l}600- \\
1050\end{array}$ & $\begin{array}{l}\text { Entre los } \\
\text { puntos D- } \\
\text { E a los } 800 \\
\text { y } 850^{\circ} \mathrm{C} \\
\text { aproximad } \\
\text { amente se } \\
\text { presenta la } \\
\text { reacción } \\
\text { endotérmic } \\
\text { a, este } \\
\text { efecto } \\
\text { podría } \\
\text { correspond } \\
\text { er a la } \\
\text { deshidroxil } \\
\text { ación } \\
\text { (dilución } \\
\text { de los } \\
\text { gases } \\
\text { combustibl } \\
\text { es por el } \\
\text { agua } \\
\text { formada) } \\
\text { de la } \\
\text { arcilla que } \\
\text { se prolongarí } \\
\text { pro hasta la } \\
\text { a temperatur } \\
\text { a de } \\
\text { fusión. }\end{array}$ & $\begin{array}{l}\text { Entre los } \\
\text { puntos C- } \\
\text { D a los } \\
800 \text { y } \\
1000^{\circ} \mathrm{C} \\
\text { aproximad } \\
\text { amente se } \\
\text { presenta la } \\
\text { reacción } \\
\text { endotérmic } \\
\text { a } \\
\text { constante, } \\
\text { este efecto } \\
\text { podría } \\
\text { correspond } \\
\text { er a la } \\
\text { deshidroxil } \\
\text { ación } \\
\text { (dilución } \\
\text { de los } \\
\text { gases } \\
\text { combustibl } \\
\text { es por el } \\
\text { agua } \\
\text { formada) } \\
\text { de la } \\
\text { arcilla que } \\
\text { se } \\
\text { prolongarí } \\
\text { a hasta la } \\
\text { temperatur } \\
\text { a de } \\
\text { fusión. }\end{array}$ & $\begin{array}{l}\text { La curva } \\
\text { presenta un } \\
\text { descenso } \\
\text { gradual y } \\
\text { progresivo, } \\
\text { con curvas } \\
\text { característica } \\
\text { s de tipo III y } \\
\text { VII, con una } \\
\text { pérdida de } \\
\text { peso del } \\
0,8 \% \text { En } \\
\text { donde se } \\
\text { pudo haber } \\
\text { presentado la } \\
\text { liberación de } \\
\mathrm{CO}_{2} \text {. }\end{array}$ & $\begin{array}{l}\text { La curva } \\
\text { presenta } \\
\text { descenso } \\
\text { gradual } \\
\text { progresivo, con } \\
\text { curvas } \\
\text { características } \\
\text { de tipo VI y } \\
\text { VII, con una } \\
\text { pérdida de peso } \\
\text { del 5\%. En } \\
\text { donde se pudo } \\
\text { haber } \\
\text { presentado la } \\
\text { liberación de } \\
\mathrm{CO}_{2} \text {. }\end{array}$ \\
\hline $\begin{array}{c}1050 \\
- \\
1250\end{array}$ & $\begin{array}{lr}\text { Entre } & \text { los } \\
\text { puntos } & \text { F- } \\
\text { G, } & \text { se }\end{array}$ & $\begin{array}{ll}\text { Entre } & \text { los } \\
\text { puntos } & \text { D- } \\
\text { E, } & \text { se }\end{array}$ & $\begin{array}{lr}\text { Se } & \text { presenta } \\
\text { una } & \text { curva } \\
\text { característica }\end{array}$ & $\begin{array}{l}\text { Se presenta una } \\
\text { pérdida de peso } \\
\text { del } 2,5 \% \text {. }\end{array}$ \\
\hline
\end{tabular}

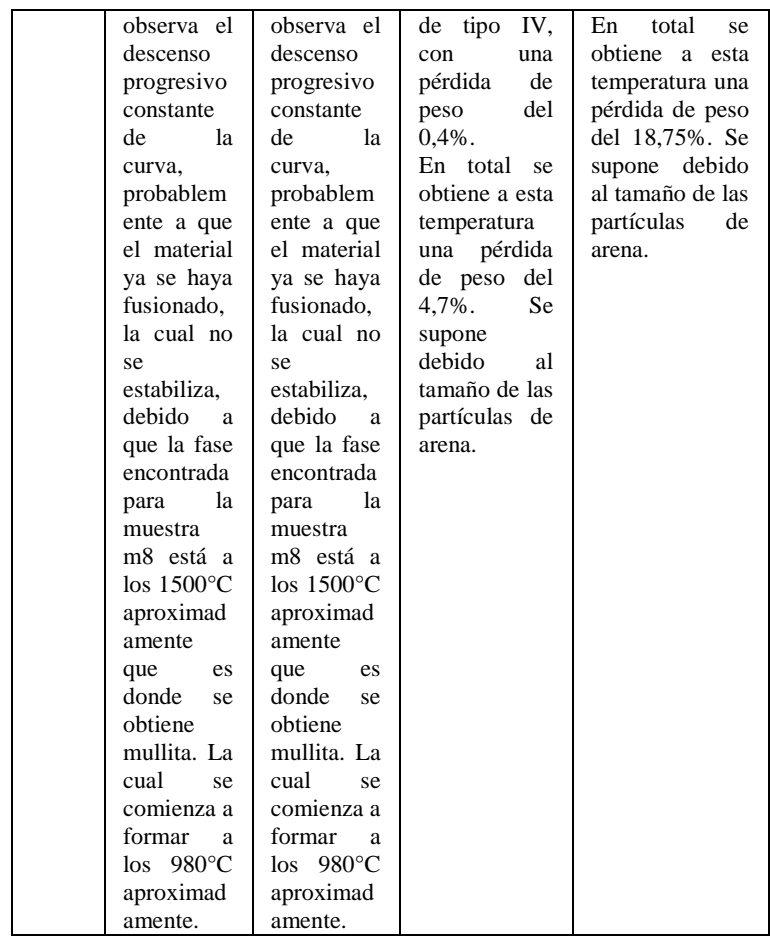

Para el caso de la mezcla m8, esta presenta un comportamiento variado en relación a la mezcla $\mathrm{m} 7$, y es debido a la cantidad de partículas en forma de arena lo cual hace que las partículas no se fusionen rápidamente generando cambios en peso, por lo que se observa que a temperaturas superiores a $1000^{\circ} \mathrm{C}$ el valor del peso comienza a ser constante (tabla 2).

Teniendo en cuenta que la temperatura de cocción de $950^{\circ} \mathrm{C}$ que se lleva actualmente para los productos fabricados por la empresa, se observa un comportamiento adecuado, dado a que esta temperatura comienza a ser constante para encontrar la fase de mullita.

El porcentaje de contracción lineal de las mezclas después del tratamiento de cocción fueron de 6,059 $\pm 0,25$ y $4,101 \pm 0,19$ para la mezcla $\mathrm{m} 7$ y $\mathrm{m} 8$ respectivamente. Los anteriores resultados son comparables con los que se evidencian en la literatura evaluada.

Las contracciones en cocido para la arcilla son elevadas y tienen un marcado aumento a partir de los $1050^{\circ} \mathrm{C}$, sin terminar de contraer a $1200^{\circ} \mathrm{C}$. Este comportamiento es típico en materiales de características arenosas (Barba et al., 2004).

Los resultados obtenidos son de gran importancia teniendo en cuenta que se estableció una posible curva ideal de temperatura, que permita evitar las 
roturas de precalentamiento, cocción o enfriamiento en el bloque.

Por lo tanto, el bloque responde a las temperaturas de cocción deformándose elásticamente, la cual tiene un límite que se puede llegar a sobrepasar si el calentamiento o enfriamiento son muy rápidos. Por lo tanto la pieza queda sometida a tensión y se deforma. En otras ocasiones si dichas tensiones son superiores a la resistencia que el material, se producirán grietas de cocción. Conociendo la resistencia y elasticidad de la pieza a cada temperatura de cocción, se podría establecer una velocidad de calentamiento tal que las tensiones derivadas de los factores señalados fuesen siempre inferiores a la resistencia del bloque. Actualmente las investigaciones sobre materiales cerámicos, no poseen datos precisos sobre los valores apuntados acerca del comportamiento de las arcillas en todo su proceso productivo.

La siguiente figura 7 muestra la curva de cocción propuesta a nivel laboratorio para la mezcla $\mathrm{m} 7$, teniendo en cuenta los análisis térmicos:

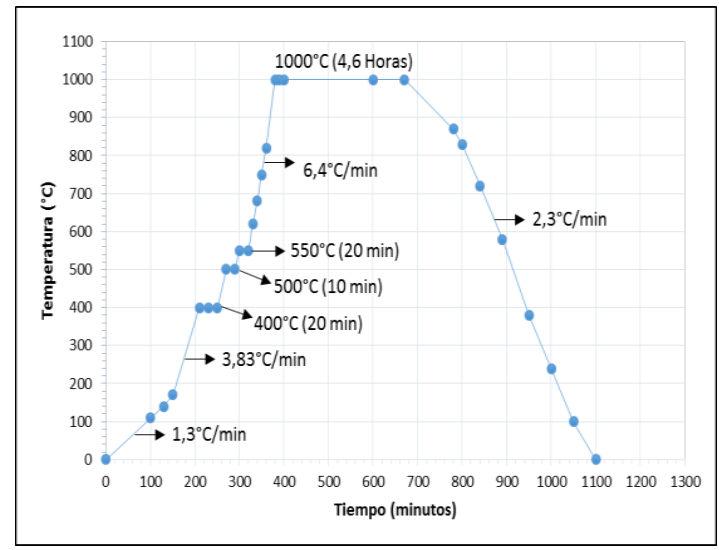

Figura 7. Curva de quema óptima propuesta para el horno de la empresa.

Se propuso que durante la etapa de precalentamiento en el inicio del ciclo térmico alcanzará una temperatura de $170^{\circ} \mathrm{C}$ con una velocidad de calentamiento aproximadamente $1,3^{\circ} \mathrm{C} / \mathrm{min}$ de 2,5 horas para ayudar a la perdida de agua higroscópica (evaporación del agua) y evitar fisuras debido a que las muestras poseen partículas grandes de arena.

Luego cuando ya obtiene la temperatura de $170^{\circ} \mathrm{C}$, es la etapa donde se ha evaporado la humedad residual, desde este punto se propone una velocidad de calentamiento de $3,83^{\circ} \mathrm{C} / \mathrm{min}$ hasta alcanzar una temperatura de $400^{\circ} \mathrm{C}$ donde permanecerá por un tiempo de 20 minutos
(Evaporación de agua ligada), luego para alcanzar una temperatura de $550^{\circ} \mathrm{C}$ con una velocidad de $2,5^{\circ} \mathrm{C} / \mathrm{min}$ con la finalidad de descomponer $\mathrm{y}$ disociar los hidróxidos de hierro.

Luego para hasta alcanzar los $1000^{\circ} \mathrm{C}$ se propone una velocidad de calentamiento de $6,4^{\circ} \mathrm{C} / \mathrm{min}$ debido a que a $\operatorname{los} 573^{\circ} \mathrm{C}$ se produce una reacción exotérmica relacionada con la transformación alotrópica del cuarzo $\alpha$ a $\beta$, con la la curva característica tipo VII.

Posteriormente, se propuso una temperatura ideal de $1000^{\circ} \mathrm{C}$ que es donde se estabilizan las curvas ATD y ATG para la mezcla $\mathrm{m} 7$ donde se mantendrá por 4,6 horas para garantizar la fusión de las partículas y obtener a temperaturas de aproximadamente $1500^{\circ} \mathrm{C}$ la fase de mullita para unos bloques con características refractarias con mejores propiedades.

Finalmente en la etapa de enfriamiento se propuso una velocidad de $2,3^{\circ} \mathrm{C} / \mathrm{min}$ en un tiempo de aproximadamente 7,2 horas, con la finalidad de que el producto no tenga choques térmicos $\mathrm{y}$ por consiguiente fisuras y deformaciones.

Teniendo en cuenta lo anterior, los productos de la industria cerámica se pueden clasificar según varios criterios, separados en dos grupos: De pasta porosa, los cuales son cosidos a menos de $1200^{\circ} \mathrm{C}$ y los de pasta compacta los cuales son cocidos a más de $1200^{\circ} \mathrm{C}$ para obtener una fusión que aglomere todas las partículas de la mezcla. Por lo que se concluye que el horno para la cocción de la empresa no alcanza una curva con la cual se garantice la calidad del producto final.

\section{CONCLUSIONES}

El proceso de cocción de la empresa estudiada no cuenta con la instrumentación y control que le permita el registro de datos de temperatura, suministro de aire y carbón, lo que no permite mejorar las condiciones de dosificación manual adicionalmente rastrear problemas particulares del horno en cada disminución de la temperatura no evidenciada.

Las mezclas estudiadas ( $\mathrm{m} 7 \mathrm{y} \mathrm{m} 8$ ) no presentan contracciones normales, debido que se consideran optimas a aquellas que no superen el 2,0\% a temperaturas hasta $970^{\circ} \mathrm{C} \mathrm{y} \mathrm{a} 1050^{\circ} \mathrm{C}$ no mayores al 4,0\% para arcillas utilizadas en la fabricación de productos extruidos. 
Se puede afirmar que una buena arcilla para ser usada en la producción de productos de mampostería aquellas muestras que funcionan a bajas temperatura, es decir entre $950^{\circ} \mathrm{C}$ y $1050^{\circ} \mathrm{C}$. En donde además, presentan valores de absorción de agua que cumplen con la norma de producto terminado que se quiere producir, como lo establece la norma NTC 4017 y 4205.

Con la finaldiad de tener la seguridad de cumplir con lo exigido por la norma técnica, es necesario establecer cocciones a temperaturas entre los $1000^{\circ} \mathrm{C}$ y $1050^{\circ} \mathrm{C}$, donde se dejan de producir reacciones exotérmicas producto de los componentes de las muestras. Donde evidentemente se debe disminuir el tamaño de partícula mediante molienda o tamizado, con lo que evidentemente los poros del material se cierren más fácilmente con la temperatura que alcancen en el horno.

\section{RECONOCIMIENTO}

Artículo de investigación científica derivado del proyecto de investigación "caracterización físico química de las arcillas utilizadas en la fabricación de productos cerámicos en una ladrillera de Ocaña Norte de Santander", financiado por la "Universidad Francisco de Paula Santander, Ocaña" con código: 158-08-021, realizado en el grupo de investigación INGAP. Fecha de inicio: 07/2016; Fecha de finalización: 07/2017.

\section{REFERENCIAS}

Amado, J. D. S., Villafrades, P. Y. M., \& Tuta, E. M. C. (2011). Caracterización de arcillas y preparación de pastas cerámicas para la fabricación de tejas y ladrillos en la región de Barichara, Santander. DYNA (Colombia), 78(167), 50-58. Retrieved from http://www.scopus.com/inward/record.url?ei $\mathrm{d}=2$-s 2.0 83155172913\&partnerID=tZOtx3y 1

Araque, J. A., Díaz, J. L., \& Gualdrón, O. (2012). Optimización del THD en un covertidor multinivel monofásico usando algoritmos genéticos. Revista Colombiana de Tecnologías de Avanzada, 1(21), 60-66.

Barba, A., Beltrán, V., Feliu, C., Garcia, J., Gines, F., Sánchez, E. Y., \& Sanz, V. (2004). Manual para el control de la calidad de materias primas arcillosas (Instituto). Castellon, España.
Barranzuela, J. (2014). Proceso productivo de los ladrillos de arcilla producidos en la región piura. Universidad de Piura.

Carriazo, J., Molina, R., \& Moreno, S. (2008). Caracterización Térmica y Espectroscópica de Arcillas Pilarizadas con Soluciones Polihidroxocatiónicas de Al, Ce, Fe. Revista Colombia de Química, 37(2), 189-202.

Cely-Illera, L. (2016). Raw materials for the ceramics industry from norte de santander. I. Mineralogical, chemical and physical characterization. Revista Facultad de Ingeniería Universidad de Antioquia, (80), 31-37. http://doi.org/10.17533/udea.redin.n80a04

Cely Illera, L., \& Bolívar León, R. (2015). Materia prima para la industria cerámica de Norte de Santander . II . Evaluación del comportamiento térmico y su incidencia en las propiedades tecnológicas. Respuestas, 20(1), 84-94.

Douglas, A., Skoog, F., Holler, J., \& Timothy A, N. (2001). Principios de análisis instrumental $5^{a}$ edición. (McGraw-Hil). Interamericana de España.

Florez Arenas, A. (2015). Caracterización de arcillas y preparación de pastas cerámicas para la fabricación de ladrillos en la ladrillera Mariscal Robledo S.A. Facultad de Tecnologías: Química Industrial, Universidad Tecnológica de Pereira, 1-19.

Flórez Solano, E., García-León, R. A., \& Escrobar Macea, M. (2017). Modelo de equipo de prensado tipo palanca, para mejorar la producción de queso en la provincia de Ocaña. Revista Colombiana de Tecnologías de Avanzada, 2(28), 140-149.

García-León, R. A., \& Bolívar León, R. (2017). Caracterización Hidrométrica de las Arcillas Utilizadas en la Fabricación de Productos Cerámicos en Ocaña, Norte de Santander. INGE CUC, 13(1), 1-9. http://doi.org/http://dx.doi.org/10.17981/inge cuc.13.1.2017.05

García-León, R. A., Bolívar León, R., \& Flórez Solano, E. (2016). Validación de las propiedades físico-mecánicas de bloques $\mathrm{H}$ 10 fabricados en Ocaña Norte de Santander y la región. Ingenio UFPSO, 10(2011-642X), $17-26$.

García-León, R. A., \& Flórez Solano, E. (2016). Determinación de la ventana del proceso productivo en la fabricación de bloques $\mathrm{H}-10$ en Ocaña Norte de Santander y la región. Ingenio UFPSO, 9(2011-642X), 35-43.

García-León, R. A., Flórez Solano, E., \& Acosta 
Pérez, M. A. (2015). Análisis estructural de una máquina prensadora para producción de ladrillo macizo para las pequeñas industrias artesanales de materiales cerámicos en Ocaña Norte de Santander y en la región. Revista Colombiana de Tecnologías de Avanzada, 1(1692-7257), 7.

Guerrero Gomez, G., Marrugo Carreazo, D., \& Gomez Camperos, J. (2015). Desarrollo de instrumento virtual enfocado en la adquisición de datos para generar perfiles de temperatura en hornos. Ingenio UFPSO, 8(2011-642X), 47-58.

Jácome Manzano, S. A. (2012). Evaluación termodinámica del proceso de cocción y análisis de gases en hornos a cielo abierto y Hoffman en Ocaña (Universida). Ocaña: Repositorio ufpso.

Linseis. (2012). Thermal Analysis.

Mejía, A., Augusto, C., Cortés, P., Ivan, C., \& Jaimes, R. (2016). Máquina clasificadora de flores: Diseño y construcción. Revista Colombiana de Tecnologías de Avanzada, 1(27), 40-45.

Moreno Rubio, J. J., Jimenez López, A. F., \& Barrera Lombana, N. (2013). El amplificador de potencia de carga sintonizada. Revista Colombiana de Tecnologías de Avanzada, 2(22), 9-13.

Muñoz Meneses R. A., Muñoz Chaves J.A., Mancilla P, R. P. J. E. (2007). Caracterización fisicoquímica de arcillas del municipio de Guapi- costa pacífica caucana (Colombiana). Química, 31, 537-544.

Perales López, N. J.;, \& Barrera Vargas, M. (2013). Análisis estructural por DRX de una arcilla natural Colombiana modificada por pilarización. Revista de Investigaciones Universidad Del Quindio, 24(1), 100-106.

Ramírez Calderón, Y. E. (2012). Determinación de las fases presentes en pisos y revestimientos cerámicos a base de arcilla cocidos a diferentes temperaturas y su influencia en las propiedades técnicas. Universidad Nacional de Colombia.

Rendon Ríos, C. M. (2009). Uso de materias primas colombianas para el desarrollo de baldosa cerámicas con alto grado de gresificación. Universidad Nacional de Colombia sede Medellin.

Riojas Castillo, O., \& Rodríguez Montaña, N. E. (2004). Características de hornos para productos cerámicos del Parque Minero Industrial El Mochuelo, localidad 19 de Bogotá, D.C. Con-Ciencias.

Rodríguez, T. V. (2007). Los minerales industriales en Castilla y León. Junta de Castilla y León (Domènech e, Vol. 1). España.

http://doi.org/10.1109/EEM.2011.5953081

Sáenz García, H. A. (2014). Caracterización fisicoquímica de ultrafinos de arcillas y su efecto sobre comportamiento reológico. Universidad Nacional de Colombia.

Sanchez, L. (2016). Tema 6. Análisis térmico: TG y DTA. Retrieved from http://www.uco.es/ iq2sagrl/TranspATG.pdf

Velasco Sánchez, E., Sánchez Lozano, M., Peral Orts, R., \& AME, G. (2016). Libro de articulos del XXI Congreso Nacional de Ingeniería Mecánica (Universita). Elche. España.

Zuluaga Castrillón, D., Henao Arrieta, A. P., García Palacio, D. F., Rodríguez, J. E., Hoyos Machado, Á. M., López, M. E., \& Gómez Álvarez, C. (2016). Caracterización térmica, química y mineralógica de un tipo de arcilla roja propia de la región andina Colombiana, empleada para la producción de ladrillos para construcción. Revista Colombiana de Materiales, (9), 53-63. 